\title{
Morphometric Study of Upper End of Tibia in Dakshina Kannada Population
}

\author{
Chaitra D. ${ }^{1}$, Divya Pai², Pretty Rathnakar ${ }^{3}$, Remya K. ${ }^{4}$ \\ ${ }^{1}$ Department of Anatomy, K.S. Hegde Medical Academy, Deralakatte, Mangalore, Karnataka, India. \\ ${ }^{2}$ Department of Anatomy, K.S. Hegde Medical Academy, Deralakatte, Mangalore, Karnataka, India. \\ ${ }^{3}$ Department of Anatomy, K.S. Hegde Medical Academy, Deralakatte, Mangalore, Karnataka, India. \\ ${ }^{4}$ Department of Anatomy, K.S. Hegde Medical Academy, Deralakatte, Mangalore, Karnataka, India.
}

\section{ABSTRACT}

\section{BACKGROUND}

The knee joint is a compound synovial joint which transmits the body weight and also helps in movement. We wanted to study the morphological features of tibia, that is condylar and intercondylar surface with regard to south Indian population. The data obtained from this study can be useful for assessing the knee deformity. The morphometric parameters of upper end of tibia can be used to guide treatment and monitor outcome of total knee replacement surgeries.

\section{METHODS}

The present study was carried out on 75 dry tibias [32 right and 43 left] of unknown sex, in the department of anatomy K.S. Hegde Medical Academy, Deralakatte, Mangalore. Measurements were taken by using the digital vernier caliper. Statistical analysis was done using SPSS software.

\section{RESULTS}

The morphometric significance of eight variables, namely, antero-posterior diameter of medial and lateral tibial condyle, transverse diameter of medial an lateral tibial condyle, antero-posterior diameter of intercondylar area, antero-posterior diameter of anterior and posterior intercondylar area was recorded. The mean AP diameter of right medial tibial condyle $(39.51 \pm 3.2)$ is more than the left medial condyle (38.95 \pm $4.5)$. But the mean transverse measurement of left tibial condyle $(27.24 \pm 2.7)$ is slightly more than the right medial tibial condyle $(26.35 \pm 2.8)$. The mean value of transverse measurement of right lateral condyle $(24.71 \pm 2.4)$ is less than the left lateral condyle $(25.39 \pm 3.2)$. The results obtained were compared with the previous studies.

\section{CONCLUSIONS}

The conventional prosthesis available in the market are designed for the Caucasians. When compared to the Caucasians, south Indian population have smaller anatomical measurements. By using the measurements and proper guidelines, knee prosthesis best suited for south Indian population can be designed. This also ensures long term utilization of prosthesis.

\section{KEY WORDS}

Tibial Condyles, Intercondylar Area, Knee Joint, Knee Deformity

\author{
Corresponding Author: \\ Divya Pai, \\ Department of Anatomy, \\ K.S. Hegde Medical Academy, \\ Deralakatte, Mangalore, \\ Karnataka, India. \\ E-mail: divyas27j89@gmail.com \\ DOI: $10.14260 /$ jemds $/ 2020 / 272$ \\ Financial or Other Competing Interests: \\ None. \\ How to Cite This Article: \\ Chaitra D, Pai D, Rathnakar P, et al. \\ Morphometric study of upper end of tibia in \\ Dakshina Kannada population. J. Evolution \\ Med. Dent. Sci. 2020;9(15):1252-1255, DOI: \\ 10.14260/jemds/2020/272
}

Submission 27-12-2019,

Peer Review 21-03-2020,

Acceptance 27-03-2020,

Published 13-04-2020.

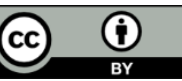




\section{BACKGROUND}

Tibia and fibula are the two bones of the leg, out of which tibia plays an important role in transmission of weight. Tibia is second longest bone of the human body. It represents the preaxial bone of the leg. The most of the bone is subcutaneous and palpable. The upper end of tibia is expanded to form medial and lateral condyle which articulates with the lower expanded condyles of femur to form condylar variety of synovial joint. Both the condyles are separated by an intercondylar area. The intercondylar area is rough, narrowest centrally and forms an intercondyar eminence edges of which projects slightly proximally as lateral and medial intercondylar tubercles. The condyles of tibia and femur are separated by fibrocartilaginous menisci. The medial condyle is larger than the lateral condyle. It bears an articular surface which is concave and extend on a medial intercondylar tubercle.

This is in conformity with the medial femoral condyle and the meniscus. Below this is a small circular facet for articulation wih upper end of fibula. Its proximal articular surface for the corresponding femoral condyle is almost circular, centrally concave and extends on to a lateral intercondylar tubercle on the medial aspect. The knee joint is a compound joint which brings out the significant function of adjusting the center of body mass and posture, requiring for the great range of movement in three dimensions together with capability to bear high forces.[1],[2] Tibia is also known as shinbone, which is stronger and larger of the two bones of leg. The proximal end of tibia is a crucial component of the knee joint, through the tibio-femoral articulation plays an important role in the conduction of body weight from the femur above to the talus below and is therefore unique in day to day function. [2]

The articular surfaces are covered by hyaline cartilage. The morphology of lateral tibial condyle differs from that of medial tibial condyle. The medial condyle is larger, ovoid and lateral condyle is small and circular.[3] The morphometric of upper end of tibia is vital as it provides reliable method of assessing knee deformity. Knee replacement surgery is most commonly performed in people with advanced osteoarthritis. To correct significant knee joint trauma in young patients, total knee replacement is an option. Total knee arthroplasty and unicompartmental knee arthroplasty are both precision surgery. It requires accuracy in prosthesis sizing to ensure a successful outcome.

The morphometric measurements of upper end of tibia helps in reconstructive surgical procedures in orthopaedic practice. This study is beneficial for orthopaedic surgeon in understanding the anatomical morphology of the upper end of tibia as well as forensic expert and physical anthropologist. The morphometric data of upper end of tibia can be used to guide treatment and monitor outcome of total knee replacement surgeries. ${ }^{[4]}$ This study provides guidelines for designing tibial component for unilateral knee arthroplasty for the said population. Hence, the study of dimensions of upper end of tibia is applicable in knee joint surgeries, which requires various interventions in numerous pathological and degenerative conditions of knee joint.

\section{METHODS}

The present study was carried out on 75 dry tibias [32 right and 43 left] of unknown sex, in the department of anatomy K.S. Hegde Medical academy, Deralakatte, Mangalore. All the measurements were recorded with the help of digital vernier caliper. The following parameters were studied on the proximal end of the tibia-

a) Antero-Posterior (AP) diameter of medial tibial condyle: it represents the distance between anterior and posterior borders of superior articular surface of medial condyle )

b) Transverse diameter of medial tibial condyle: it represents maximum transverse diameter of superior articular surface of medial condyle

c) AP diameter of lateral tibial condyle: it represents the distance between anterior and posterior borders of superior articular surface of lateral condyle

d) Transverse diameter of lateral tibial condyle: it represents maximum transverse diameter of superior articular surface of lateral condyle

e) AP diameter of intercondylar area: it represents maximum distance between anterior and posterior borders (table 3)

f) Transverse diameter of intercondylar area: it represents maximum transverse diameter at following three levelsanterior end, middle narrow part and posterior part.

g) AP diameter of anterior intercondylar area: it represents the maximum distance between anterior border and intercondylar eminence

h) AP diameter of posterior intercondylar area: it represents the maximum distance between intercondylar eminence and posterior border

\section{Statistical Analysis}

The morphometric measurement is taken by using a digital vernier caliper. The measurement obtained is tabulated and statistical analysis is done by using SPSS software. The results are discussed below.

\section{RESULTS}

The morphometric significance of eight variables were taken, which included AP diameter of medial tibial condyle, Transverse diameter of medial tibial condyle, AP diameter of lateral tibial condyle, Transverse diameter of lateral tibial condyle, AP diameter of intercondylar area, Transverse diameter of intercondylar area, AP diameter of anterior intercondylar area, AP diameter of posterior intercondylar area. The measurements obtained are tabulated in the tables. It is observed that the mean AP diameter of right medial tibial condyle $(39.51 \pm 3.2)$ is more than the left medial condyle (38.95 \pm 4.5$)$. But the mean transverse measurement of left tibial condyle $(27.24 \pm 2.7)$ is slightly more than the right medial tibial condyle (26.352.8). The mean value of transverse measurement of right lateral condyle $(24.71 \pm 2.4)$ is less than the left lateral condyle $(25.39 \pm 3.2)$. The transverse measurement of intercondylar area was measured at the levels of anterior end, middle narrow part \& posterior end of intercondylar area are tabulated in table 4. Except for the transverse measurement of posterior end of intercondylar 
area of left tibiae, the transverse measurement of intercondylar area of anterior end, middle narrow part of left tibia is more than the right tibiae. The AP measurement of anterior-posterior intercondylar area of right side tibiae is less than the left side tibiae. The mean and standard deviation values are tabulated in table 5 .

\begin{tabular}{|c|c|c|c|}
\hline Medial Condyle & Mean & SD & SEM \\
\hline Right (AP) & 39.510 & 3.236 & 0.572 \\
\hline Right (transverse) & 26.357 & 2.812 & 0.497 \\
\hline Left (AP) & 38.951 & 4.560 & 0.695 \\
\hline Left (transverse) & 27.248 & 2.757 & 0.420 \\
\hline \multicolumn{4}{|c|}{ Table 1. AP and Transverse Measurements of Medial Tibial Condyl } \\
\hline \multicolumn{4}{|c|}{$p$ value: $[p=0.556], p$ value: $[p=0.174]$} \\
\hline Lateral Condyle & Mean & SD & SEM \\
\hline Right (AP) & 33.572 & 3.065 & 0.542 \\
\hline Right (transverse) & 24.710 & 2.491 & 0.440 \\
\hline Left (AP) & 32.865 & 3.004 & 0.458 \\
\hline Left (transverse) & 25.394 & 3.264 & 0.498 \\
\hline \multicolumn{4}{|c|}{$\begin{array}{l}\text { Table 2. Mean Value of AP and Transverse Measurement } \\
\text { of Lateral Condyle }\end{array}$} \\
\hline \multicolumn{4}{|c|}{$p$ value $[p=0.321], p$ value $[p=0.326]$} \\
\hline
\end{tabular}

\begin{tabular}{|c|c|c|c|}
\hline Intercondylar Area & Mean & SD & SEM \\
\hline Right & 40.503 & 3.655 & 0.646 \\
\hline Left & 41.058 & 4.139 & 0.631 \\
\hline \multicolumn{3}{|l|}{ Table 3. AP Measurement of Intercondylar Areas } \\
\hline $\mathrm{p}$ value [p=0.549]
\end{tabular}

\begin{tabular}{|c|c|c|c|c|}
\hline Intercondylar Area & & & & \\
\hline Transverse[anterior end] & & Mean & SD & SEM \\
\hline & Right & 20.429 & 3.625 & 0.641 \\
\hline Transverse [middle narrow part] & Right & 20.781 & 3.705 & 0.565 \\
\hline & Left & 9.922 & 1.840 & 0.325 \\
\hline Transverse [posterior part] & Right & 15.488 & 2.813 & 0.259 \\
\hline \multicolumn{6}{|c|}{ Left } & 14.520 & 2.189 & 0.497 \\
\hline Table 4. Transverse Measurement of Intercondylar Area at the Levels of \\
Anterior End, Middle Narrow Part, \& Posterior End of Intercondylar Area. \\
\hline
\end{tabular}

\begin{tabular}{|l|c|c|c|c|c|}
\hline \multirow{3}{*}{ Anterior Intercondylar Area } & Side & Mean & S D & SEM & p \\
\cline { 2 - 6 } & Right & 27.333 & 3.043 & 0.538 & \multirow{2}{*}{0.328} \\
\cline { 2 - 6 } & Left & 27.500 & 2.962 & 0.452 & \\
\hline \multirow{3}{*}{ Posterior Intercondylar Area } & & & & & \\
\cline { 2 - 6 } & Right & 19.410 & 1.933 & 0.342 & \multirow{2}{*}{0.342} \\
\cline { 2 - 5 } & Left & 19.885 & 2.158 & 0.329 & \\
\hline
\end{tabular}

Table 5. Comparison of Measurement of Intercondylar Area $\mathrm{p}$ value [ $\mathrm{p}=0.328] \mathrm{p}$ value [ $\mathrm{p}=0.342]$

\section{DISCUSSION}

The current study establishes the exact value for the morphometric attributes of upper end of tibia in south Indian population. The study was done using $\mathrm{t}$-test and values were tabulated. The data obtained in the present study will provide a basis for designing the optimal tibial component for unilateral and total knee arthroplasty. Servien et al,[4] had measured anteroposterior measurements of medial and lateral condyle in French population and found it to be $50.8+$ $3.3 \mathrm{~mm}$ and $47.2+3.3 \mathrm{~mm}$ respectively. The study however was independent of sex and side. However, in the present study the AP measurement of medial condyle of both sides and lateral condyle was found to be $39.51+3.23 \mathrm{~mm}, 38.95+4.56$ $\mathrm{mm}$ and $33.57+3.06 \mathrm{~mm}, 32.86+3.00 \mathrm{~mm}$. In the present study, the transverse measurement of medial condyle of both the sides were found to be $26.35+2.81 \mathrm{~mm}, 27.24+2.75 \mathrm{~mm}$ and $24.71+2.49 \mathrm{~mm}, 25.39+3.26 \mathrm{~mm}$ respectively. In a study done by Kwak et al,[5] had measured AP measurement of intercondylar area in Korean population but irrespective of side and sex. Their values were found to be $47.3+3.8 \mathrm{~mm}$. In the present study, we found the values to be $40.50+3.65 \mathrm{~mm}$ and $41.05+4.13 \mathrm{~mm}$. In a study done by Jacobsen et al, ${ }^{[6]}$ had measured transverse measurements of intercondylar area at different levels in Danish population irrespective of sex and side. In the present study the measurement of the intercondylar area on the right and left side were found to be anterior: $20.42+3.62 \mathrm{~mm}$ and $20.78+3.70 \mathrm{~mm}$, middle: 9.54 $+1.84 \mathrm{~mm}$ and $9.92+1.69 \mathrm{~mm}$, posterior: $15.48+2.81 \mathrm{~mm}$ and $14.52+2.18 \mathrm{~mm}$. In a study done by Ankur et al, the medial condylar anteroposterior distance of superior articular surface (MCAPD) in males was $44.43 \pm 2.06 \mathrm{~mm}$ while in females it was $39.71 \pm 2.03 \mathrm{~mm}$ which was significantly higher in male than female ( $p<0.001)$. The lateral condylar anteroposterior distance of superior articular surface (LCAPD) in males was $38.38 \pm 2.39 \mathrm{~mm}$ while in females it was $35.50 \pm$ $2.37 \mathrm{~mm}$ which was significantly higher in male than female $(p<0.001)$.These measurements were lower than Iranian, Chinese, Caucasian, Brazilian and North Indian populations but higher than South Indian population. Jacobsen emphasized that the shape of posterior contours of the tuberculum mediale and laterale can be used for the identification of the posterior contours of the two tibial condyles and the posterior contour of the condylus lateralis tibiae may be identified by tracing the posterior curved part of the tuberculum laterale. According to him, the latter contour was most useful as a landmark in measuring the "drawer sign" in the knee joint by the radiological method of Kennedy and Fowler. Knee osteoarthritis is one of the most common causes of disability in adults. The damage results from a complex interplay of joint integrity, biochemical processes, genetics and mechanical forces. Osteoarthritis is the most common indication for total knee arthroplasty. Total and unicompartmental joint replacements have become the gold standard for treatment of osteoarthritis and other degenerative disorders of knee.[7]

There are several studies done on western population. So, the prosthesis designed is based on the western Caucasian population. ${ }^{[8]}$ Total knee arthroplasty and UKA are both meticulous surgeries which necessitate the precision in the prosthesis sizing to ensure an effective result as well as long term survival of the same. Suitable prosthetic design is crucial to restore the normal function in patients postoperatively. ${ }^{[9]}$ Inadequate tibial coverage can lead to tibial implant collapse because of the load being shifted to cancellous bone instead of cortical bone. Designing of prosthesis considering the gender difference have been advocated in Chinese population. ${ }^{[10]}$ Surendra et al showed a progressive decline in the condylar aspect ratio with the increase in the AP dimension, irrespective of gender[11] Westrich et al suggested that asymmetrical smaller lateral condylar surface produces better outcome compared with the symmetrically constructed prosthesis.[12]

There are some studies done on measuring the upper end of tibia based on CT scan and MRI. But these methods of measuring is not precise.[13] The present study could provide the basis for designing the optimal tibial prosthesis in total knee arthroplasty for south Indian population. The purpose of this study is to analyses the dimensions of each tibial plateau for planning of unicompartmental knee arthroplasty and to compare the morphometric data with other studies in different populations. Thus the results of this study will be 
extremely beneficial in designing appropriate size matched components of knee prosthesis for Indian population.

\section{CONCLUSIONS}

The conventional prosthesis available in the market are designed for the Caucasians. When compared to the Caucasians, south Indian population have smaller anatomical measurements. By using the measurements and proper guidelines, knee prosthesis best suited for south Indian population can be designed. This also ensures long term utilization of prosthesis.

\section{REFERENCES}

[1] Standring S. Gray's Anatomy. The anatomical basis of clinical practice. $3^{\text {th }}$ edn. Elsevier/ Churchill Livingstone 2005: p. 1436-7.

[2] Gupta C, Kumar J, Kalthur SG, et al. A morphometric study of the proximal end of the tibia in South Indian population with its clinical implications. Saudi Journal of Sports Medicine 2015;15(2):166-9.

[3] Servien E, Saffarini M, Lustig S, et al. Lateral versus medial tibial plateau: morphometric analysis and adaptability with current tibial component design. J Knee Surg Sports Traumatol Arthrosc 2008;16(12):1141-5.

[4] Gandhi S, Singla RK, Kullar JS, et al. Morphometric analysis of upper end of tibia. Journal of Clinical and Diagnostic Research 2014;8(8):AC10-AC3.
[5] Kwak DS, Surendran S, Pengatteeri YH, et al. Morphometry of the proximal tibia to design the tibial component of total knee arthroplasty for the Korean population. Knee 2007;14(4):295-300.

[6] Jacobsen K, Mehta S, Kalra R. Area intercondylaris tibiae: osseous surface structure and its relation to soft tissue structures and applications to radiography. J Anat 1974;117(Pt 3):605-18.

[7] Ogilvie-Harris DJ, Fitsialos DP. Arthroscopic management of the degenerative knee. Arthroscopy 1991;7(2):151-7.

[8] Murlimanju BV, Purushothama C, Srivastava A, et al. Anatomical morphometry of the tibial plateau in South Indian population. Italian Journal of Anatomy and Embyology 2016;121(3):258-64.

[9] Chaichankul C, Tanavalee A, Itiravivong P. Anthropometric measurements of knee joints in Thai population: correlation to the sizing of current knee prostheses. The Knee 2011;18(1):5-10.

[10] Cheng FB, Ji XF, Lai $Y$, et al. Three dimensional morphometry of the knee to design the total knee arthroplasty for Chinese population. The Knee 2009;16(5):341-7.

[11] Surendran S, Kwak DS, Lee UY, et al. Anthropometry of the medial tibial condyle to design the tibial component for unicondylar knee arthroplasty for the Korean population. Knee Surg Sports Traumatol Arthrosc 2007;15(4):43642.

[12] Westrich GH, Haas SB, Insall JN, et al. Resection specimen analysis of proximal tibial anatomy based on 100 total knee arthroplasty specimens. J Arthroplasty 1995;10(1):47-51.

[13] Anderson AF, Anderson CN, Gorman TM, et al. Radiographic measurements of the intercondylar notch: are they accurate? Arthroscopy 2007;23(3):261-8. 